\title{
Electron-transfer chain catalysis in phosphine replacement reaction: Determination of relative donor capability of arylpyridylphosphines
}

\author{
Olalere G. Adeyemi ${ }^{\text {a,b,* }}$, Ling-Kang Liu ${ }^{\text {a,c }}$ \\ ${ }^{a}$ Institute of Chemistry, Academia Sinica, Nankang, Taipei 11529, Taiwan, ROC \\ ${ }^{\mathrm{b}}$ Department of Chemical Sciences, College of Natural Sciences, Redeemer's University, Km 46, Lagos/Ibadan Expressway, Redemption City, Nigeria \\ ${ }^{\mathrm{c}}$ Department of Chemistry, National Taiwan University, Taipei 10767, Taiwan, ROC
}

Received 16 August 2006; received in revised form 4 December 2006; accepted 8 December 2006

Available online 20 January 2007

\begin{abstract}
The slight differences in the donor capabilities of $\mathrm{PPh}_{n} \mathrm{Py}_{3-n}(n=0-3)$ could be measured directly in the equilibrium of phosphine replacement reaction on $\left(\eta^{5}-\mathrm{C}_{5} \mathrm{H}_{5}\right) \mathrm{Fe}(\mathrm{CO})_{2}$-phosphine complexes, taking advantage of the radical pathway to establish equilibrium rapidly. The simultaneous determination of equilibrium constants is done in a single experiment. The donor capability increases in the order $\mathrm{PPh}_{3}<\mathrm{PPh}_{2} \mathrm{Py}<\mathrm{PPhPy}_{2}<\mathrm{PPy}_{3}$ with quantified $\left(\eta^{5}-\mathrm{C}_{5} \mathrm{H}_{5}\right) \mathrm{Fe}(\mathrm{CO})_{2}{ }^{+}$-affinity scales at $1,4.90,11.0$, and 20.3 , respectively.
\end{abstract}

(C) 2006 Elsevier B.V. All rights reserved.

Keywords: Electron-transfer chain catalysis; Arylpyridylphosphines; Iron complexes; Radical process; Phosphine exchange; Cyclic voltammetry

\section{Introduction}

Organophosphines are used in many metal mediated reactions [1]. As a consequence, chemists have been interested in deducing their stereo-electronic properties [2], among which the best known parameters are the cone angle $\Theta$ and the electronic parameter $\chi$ by Tolman [3]. Also a good parameter, the $\mathrm{p} K_{\mathrm{a}}$ value for $\mathrm{HPR}_{3}{ }^{+}$depends on the interaction between the phosphorus and $\mathrm{H}^{+}$, a hard acid [4]. Such an interaction is generally considered not the same as that between the phosphorus ligand and a low valent metal center in an organometallic complex. Derived from the IR data for $\mathrm{LNi}(\mathrm{CO})_{3}(\mathrm{~L}=$ phosphorus ligand), the $\chi$ values reflect a combined property of both $\sigma$-donor and $\pi$-acceptor [3]. Many other evaluations have been attempted using IR [5], NMR [6], UV/Vis [7], Photoelectron [8], and Mössbauer spectroscopy [9], as well as the-

\footnotetext{
* Corresponding author. Address: Department of Chemical Sciences, College of Natural Sciences, Redeemer's University, Km 46, Lagos/ Ibadan Expressway, Redemption City, Nigeria. Tel.: +234 8055516450.

E-mail addresses: adeyemio@run.edu.ng,drlereadeyemi@yahoo.com (O.G. Adeyemi).
}

oretical [10], and electrochemical [11] methods and X-ray crystallography [12].

Giering et al. reported in their quantitative analysis of ligand effects, a series of phosphines with $\Theta, \mathrm{p} K_{\mathrm{a}}, \chi, v_{\mathrm{CO}}$ and $E^{\circ}$ values were tabulated. In particular, the $v_{\mathrm{CO}}$ and $E^{\circ}$ values were obtained from the iron-complexes $\left(\eta^{5}\right.$ $\left.\mathrm{C}_{5} \mathrm{H}_{5}\right) \mathrm{Fe}(\mathrm{CO})(\mathrm{L}) \mathrm{C}(\mathrm{O}) \mathrm{Me}$ and $\left(\eta^{5}-\mathrm{MeC}_{5} \mathrm{H}_{4}\right) \mathrm{Fe}(\mathrm{CO})(\mathrm{L})-$ $\mathrm{C}(\mathrm{O}) \mathrm{Me}$, where $\mathrm{L}$ is a phosphorus ligand [13]. For the following triarylphosphines with $\Theta=145^{\circ}$, e.g., $\mathrm{P}(p$ $\left.\mathrm{C}_{6} \mathrm{H}_{4} \mathrm{NMe}_{2}\right)_{3}, \mathrm{P}\left(p-\mathrm{C}_{6} \mathrm{H}_{4} \mathrm{OPh}\right)_{3}, \mathrm{P}\left(p-\mathrm{C}_{6} \mathrm{H}_{4} \mathrm{Me}\right)_{3}, \mathrm{PPh}_{3}, \mathrm{P}(p-$ $\left.\mathrm{C}_{6} \mathrm{H}_{4} \mathrm{~F}\right)_{3}, \mathrm{P}\left(p-\mathrm{C}_{6} \mathrm{H}_{4} \mathrm{Cl}\right)_{3}, \mathrm{P}\left(p-\mathrm{C}_{6} \mathrm{H}_{4} \mathrm{CF}_{3}\right)_{3}$, a decrease of ligand donor capability was clearly evident and there was a nice correlation among the parameters.

The series of arylpyridylphosphines $\mathrm{PPh}_{n} \mathrm{Py}_{3-n}(n=0-3)$ are also with $\Theta$ values approximately $145^{\circ}$, yet without readily available $\mathrm{p} K_{\mathrm{a}}, \chi, v_{\mathrm{CO}}$ and $E^{\circ}$ values for the evaluation of their donor capability. The only starting point is that the ${ }^{31} \mathrm{P}$ NMR chemical shifts are more upfield with more $\mathrm{Ph}$ groups $(\delta-0.6,-1.9,-3.2$, and -4.7 with increasing number of Ph-groups), a fact not necessarily translated into increasing donor capability, however. As there are slight differences in the donor capabilities of $\mathrm{PPh}_{n} \mathrm{Py}_{3-n}$, we have found that the direct measurement of an equilibrium 
constant in the phosphine replacement reaction on $\left(\eta^{5}\right.$ $\left.\mathrm{C}_{5} \mathrm{H}_{5}\right) \mathrm{Fe}(\mathrm{CO})_{2}$-phosphine complex is possible, taking advantage of the radical pathway to establish equilibrium rapidly. A simultaneous determination of equilibrium constants is also likely so that their donor capabilities could be compared in a single experiment.

\subsection{Radical process}

Pioneered by Rich and Taube in inorganic chemistry [14], the electron-transfer chain catalysis (ETC) is applied to the preparation of organometallic complexes, utilizing the greatly enhanced reactivity of organometallic $17 \mathrm{e}$ and $19 \mathrm{e}$ radicals in comparison to their $16 \mathrm{e}$ and $18 \mathrm{e}$ analogues [15-17]. The $\mathrm{PPh}_{3}$ substitution for $\mathrm{CO}$ on $\left[\left(\eta^{5}-\mathrm{C}_{5} \mathrm{H}_{5}\right)\right.$ $\left.\mathrm{Fe}(\mathrm{CO})_{3}{ }^{+}\right]$proceeds with $17 \mathrm{e}-19 \mathrm{e}$ pathway after electrochemical or chemical initiation, for instance, and yields $\left[\left(\eta^{5}-\mathrm{C}_{5} \mathrm{H}_{5}\right) \mathrm{Fe}(\mathrm{CO})_{2} \mathrm{PR}_{3}{ }^{+}\right]$quantitatively [18]. The reaction of $1: 1\left(\eta^{5}-\mathrm{C}_{5} \mathrm{H}_{5}\right) \mathrm{Fe}(\mathrm{CO})_{2} \mathrm{I} / \mathrm{PPh}_{3}$ in THF at $-78^{\circ} \mathrm{C}$, initialized by the addition of a small amount of reducing agents, e.g., diluted $\mathrm{RLi}[19]$ or $\left(\eta^{5}-\mathrm{C}_{5} \mathrm{H}_{5}\right)_{2} \mathrm{Co}$, also follows the $17 \mathrm{e}-19 \mathrm{e}$ pathway and gives quantitative precipitates of $\left[\left(\eta^{5}-\mathrm{C}_{5} \mathrm{H}_{5}\right) \mathrm{Fe}(\mathrm{CO})_{2}\left(\mathrm{PPh}_{3}\right)^{+}\right]\left[\mathrm{I}^{-}\right]$instantaneously, $\mathrm{I}^{-}$being selectively replaced by $\mathrm{PPh}_{3}[20]$. The anion $\left[\mathrm{I}^{-}\right]$could be exchanged into $\left[\mathrm{PF}_{6}{ }^{-}\right]$without difficulty.
With easily available $\left[\left(\eta^{5}-\mathrm{C}_{5} \mathrm{H}_{5}\right) \mathrm{Fe}(\mathrm{CO})_{2}\left(\mathrm{PPh}_{3}\right)^{+}\right]$ $\left[\mathrm{PF}_{6}{ }^{-}\right]$, the $17 \mathrm{e}-19 \mathrm{e}$ pathway enables one to instantly differentiate an incoming $\mathrm{PPh}_{n} \mathrm{Py}_{3-n}$ replacement for $\mathrm{PPh}_{3}$ on $\left[\left(\eta^{5}-\mathrm{C}_{5} \mathrm{H}_{5}\right) \mathrm{Fe}(\mathrm{CO})_{2} \mathrm{PPh}_{3}{ }^{+}\right]$. Scheme 1 shows such an ETC reaction, the equilibrium constant $K$ of Eq. (1) being the product of $K_{\text {redox }}$ of Eq. (2) and $K_{\text {exchange }}$ of Eq. (3). The former is the coupling between the reduction of $\left(\eta^{5}-\mathrm{C}_{5} \mathrm{H}_{5}\right) \mathrm{Fe}(\mathrm{CO})_{2} \mathrm{P}^{+}$cation to $\left(\eta^{5}-\mathrm{C}_{5} \mathrm{H}_{5}\right) \mathrm{Fe}(\mathrm{CO})_{2} \mathrm{P} 1$. radical and the oxidation of $\left(\eta^{5}-\mathrm{C}_{5} \mathrm{H}_{5}\right) \mathrm{Fe}(\mathrm{CO})_{2} \mathrm{P} 2$ radical to $\left(\eta^{5}-\mathrm{C}_{5} \mathrm{H}_{5}\right) \mathrm{Fe}(\mathrm{CO})_{2} \mathrm{P}^{+}$cation. The latter is the coupling between an association of P2 with $\left(\eta^{5}-\mathrm{C}_{5} \mathrm{H}_{5}\right) \mathrm{Fe}(\mathrm{CO})_{2}^{\text {: }}$ radical to form $\left(\eta^{5}-\mathrm{C}_{5} \mathrm{H}_{5}\right) \mathrm{Fe}(\mathrm{CO})_{2} \mathrm{P} 2$ radical and a dissociation of $\left(\eta^{5}-\mathrm{C}_{5} \mathrm{H}_{5}\right) \mathrm{Fe}(\mathrm{CO})_{2} \mathrm{P1} \cdot$ radical to form $\mathrm{P} 1$ and $\left(\eta^{5}-\mathrm{C}_{5} \mathrm{H}_{5}\right) \mathrm{Fe}(\mathrm{CO})_{2}$ radical. Both redox and exchange are facile because of high energy radical process in nature [15].

The following is a qualitative analysis on the $K_{\text {redox }}$. If $\mathrm{P} 2$ is a better donor than $\mathrm{P} 1$, the $\mathrm{Fe}$ atom of $\left(\eta^{5}-\mathrm{C}_{5} \mathrm{H}_{5}\right) \mathrm{Fe}$ $(\mathrm{CO})_{2} \mathrm{P} 2 \cdot$ radical has a higher electron density than that of $\left(\eta^{5}-\mathrm{C}_{5} \mathrm{H}_{5}\right) \mathrm{Fe}(\mathrm{CO})_{2} \mathrm{P} 1^{\cdot}$ radical, i.e., the $\left(\eta^{5}-\mathrm{C}_{5} \mathrm{H}_{5}\right) \mathrm{Fe}$ $(\mathrm{CO})_{2} \mathrm{P} 2^{\circ}$ radical is likely to be a better reductant than the $\left(\eta^{5}-\mathrm{C}_{5} \mathrm{H}_{5}\right) \mathrm{Fe}(\mathrm{CO})_{2} \mathrm{P} 1 \cdot$ radical and one would expect that the $K_{\text {redox }}$ for Eq. (2) must be greater than 1. On the other hand, $K_{\text {exchange }}$ could also be analyzed in a qualitative way with Eq. (3) envisioned as P1 and P2 in competition for the

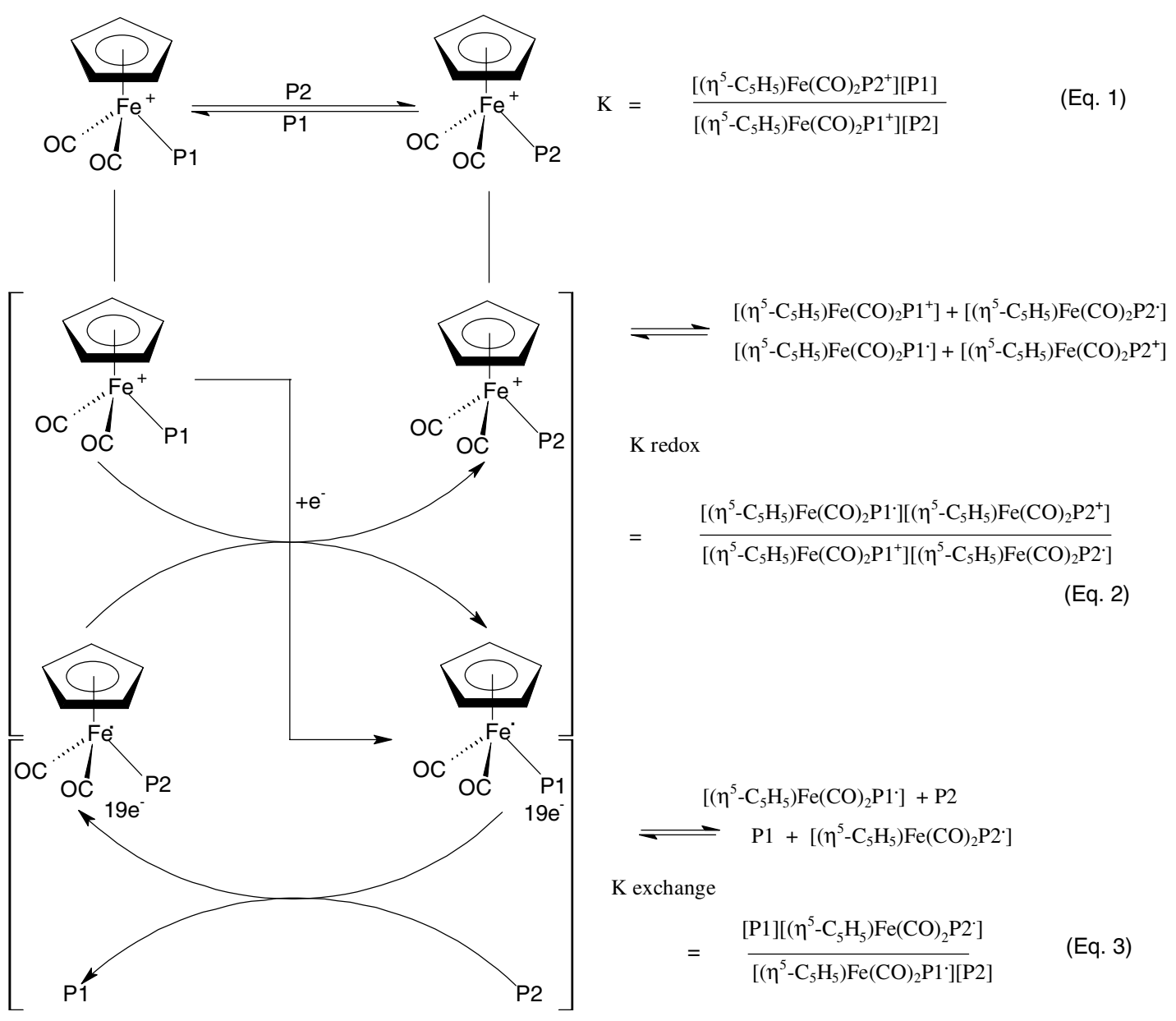

Scheme 1. Phosphine exchange reaction with an electron-transfer chain catalytic pathway. 
same $\left[\left(\eta^{5}-\mathrm{C}_{5} \mathrm{H}_{5}\right) \mathrm{Fe}(\mathrm{CO})_{2}\right.$ radical. If $\mathrm{P} 2$ is a better donor than $\mathrm{P} 1$, the $\left(\eta^{5}-\mathrm{C}_{5} \mathrm{H}_{5}\right) \mathrm{Fe}(\mathrm{CO})_{2}^{*}$ radical would prefer a ligation with $\mathrm{P} 2$ to a ligation with $\mathrm{P} 1$ and one would also expect that the $K_{\text {exchange }}$ of Eq. (3) must be greater than 1. These overall effects of a favoring phosphine substitution on the thermodynamic side combined with a rapid radical process on the dynamic side lead us to study the small difference among $\left[\left(\eta^{5}-\mathrm{C}_{5} \mathrm{H}_{5}\right) \mathrm{Fe}(\mathrm{CO})_{2} \mathrm{PPh}_{n} \mathrm{Py}_{3-n}{ }^{+}\right]$by a simple ${ }^{31} \mathrm{P}$ NMR technique, namely, the intensity integration ratio.

\section{Experimental}

\subsection{General}

All manipulations were performed under an atmosphere of prepurified nitrogen with standard Schlenk techniques. All solvents were distilled from an appropriate drying agent [21]. Infrared spectra were recorded in $\mathrm{CH}_{2} \mathrm{Cl}_{2}$ using $\mathrm{CaF}_{2}$ optics on a Perkin-Elmer 852 spectrophotometer. The ${ }^{1} \mathrm{H}$ NMR and ${ }^{13} \mathrm{C}$ NMR spectra were obtained on Bruker AC200/AC300 spectrometers, with chemical shifts reported in $\delta$ values, downfield positive, relative to the residual solvent resonance of $\mathrm{CDCl}_{3}\left({ }^{1} \mathrm{H} \delta 7.24,{ }^{13} \mathrm{C} \delta 77.0\right)$. The ${ }^{31} \mathrm{P}$ NMR spectra were obtained on Bruker AC200/AC300 spectrophotometer using $85 \% \mathrm{H}_{3} \mathrm{PO}_{4}$ as an external standard $(\delta 0.00)$. The melting points were determined on a Yanaco MPL melting-point apparatus and uncorrected. $\left(\eta^{5}-\mathrm{C}_{5} \mathrm{H}_{5}\right) \mathrm{Fe}(\mathrm{CO})_{2} \mathrm{I} \quad[22], \quad\left(\eta^{5}-\mathrm{C}_{5} \mathrm{H}_{5}\right) \mathrm{Fe}(\mathrm{CO})_{2} \mathrm{Me} \quad$ [23], $\mathrm{PPh}_{2} \mathrm{Py}$ [24], $\mathrm{PPhPy}_{2}$ [25], $\mathrm{PPy}_{3}$ [25], [ $\left(\eta^{5}-\mathrm{C}_{5} \mathrm{H}_{5}\right) \mathrm{Fe}(\mathrm{CO})_{2}-$ $\left.\mathrm{PPh}_{3}{ }^{+}\right]\left[\mathrm{PF}_{6}{ }^{-}\right][20,26]$ and $\left(\eta^{5}-\mathrm{C}_{5} \mathrm{H}_{5}\right) \mathrm{Fe}(\mathrm{CO}) \mathrm{C}(\mathrm{O}) \mathrm{Me}\left(\mathrm{PPh}_{3}\right)$ [27] were prepared according to the literature procedure. Other reagents were obtained from commercial sources and used without further purification.

\subsection{Preparation of $\left[\left(\eta^{5}-\mathrm{C}_{5} \mathrm{H}_{5}\right) \mathrm{Fe}(\mathrm{CO})_{2} \mathrm{~L}^{+}\right]\left[\mathrm{PF}_{6}^{-}\right]$,}

$L=P P h_{2} P y, P P h P y_{2}, P P y_{3}$

The procedures are the same for all $\mathrm{PPh}_{n} \mathrm{Py}_{3-n}$, following the preparation of $\left[\left(\eta^{5}-\mathrm{C}_{5} \mathrm{H}_{5}\right) \mathrm{Fe}(\mathrm{CO})_{2} \mathrm{PPh}_{3}{ }^{+}\right]\left[\mathrm{PF}_{6}{ }^{-}\right]$in the literature [20]. Only $\mathrm{L}=\mathrm{PPh}_{2} \mathrm{Py}$ is shown here as a routine run. $\left(\eta^{5}-\mathrm{C}_{5} \mathrm{H}_{5}\right) \mathrm{Fe}(\mathrm{CO})_{2} \mathrm{I}(0.86 \mathrm{~g}, 2.83 \mathrm{mmol})$ and $\mathrm{PPh}_{2} \mathrm{Py}(0.74 \mathrm{~g}, 2.83 \mathrm{mmol})$ were completely dissolved in THF $(30 \mathrm{~mL})$ and maintained at $-78^{\circ} \mathrm{C}$. A yellow precipitate appeared on addition of a trace amount of $n$-BuLi (1.6 $\mathrm{M}$ in hexane, 2 drops) to the solution. The solution was further stirred for $15 \mathrm{~min}$ before being gradually warmed up to room temperature. After filtration and washing with $\mathrm{Et}_{2} \mathrm{O}$ for several times, the precipitate together with excess $\mathrm{NH}_{4} \mathrm{PF}_{6}$ was redissolved in $\mathrm{CH}_{3} \mathrm{CN}$ with vigorous stirring. Addition of sufficient $\mathrm{H}_{2} \mathrm{O}$ gave precipitates which were filtered and then washed with $\mathrm{H}_{2} \mathrm{O}$ and $\mathrm{Et}_{2} \mathrm{O}$ to produce $\left[\left(\eta^{5}-\mathrm{C}_{5} \mathrm{H}_{5}\right) \mathrm{Fe}(\mathrm{CO})_{2} \mathrm{PPh}_{2} \mathrm{Py}^{+}\right]\left[\mathrm{PF}_{6}{ }^{-}\right]$. For $\left[\left(\eta^{5}-\mathrm{C}_{5} \mathrm{H}_{5}\right) \mathrm{Fe}(\mathrm{CO})_{2} \mathrm{PPhPy}_{2}{ }^{+}\right]\left[\mathrm{PF}_{6}^{-}\right], \quad\left(\eta^{5}-\mathrm{C}_{5} \mathrm{H}_{5}\right) \mathrm{Fe}(\mathrm{CO})_{2} \mathrm{I}$ $(0.54 \mathrm{~g}, 1.78 \mathrm{mmol}), \mathrm{PPhPy}_{2}(0.47 \mathrm{~g}, 1.78 \mathrm{mmol})$, THF $(26 \mathrm{~mL})$ and for $\left[\left(\eta^{5}-\mathrm{C}_{5} \mathrm{H}_{5}\right) \mathrm{Fe}(\mathrm{CO})_{2} \mathrm{PPy}_{3}{ }^{+}\right]\left[\mathrm{PF}_{6}{ }^{-}\right],\left(\eta^{5}-\right.$ $\left.\mathrm{C}_{5} \mathrm{H}_{5}\right) \mathrm{Fe}(\mathrm{CO})_{2} \mathrm{I} \quad(0.313 \mathrm{~g}, \quad 1.0 \mathrm{mmol}), \mathrm{PPy}_{3} \quad(0.266 \mathrm{~g}$, $1.0 \mathrm{mmol})$, THF (12 mL).
2.2.1. $\left[\left(\eta^{5}-\mathrm{C}_{5} \mathrm{H}_{5}\right) \mathrm{Fe}(\mathrm{CO})_{2} \mathrm{PPh}_{2} \mathrm{Py}^{+}\right]\left[\mathrm{PF}_{6}^{-}\right]$

Yield: $1.24 \mathrm{~g}(78 \%)$; mp: $222-223{ }^{\circ} \mathrm{C}$; IR $\left(\mathrm{CH}_{2} \mathrm{Cl}_{2}\right): v_{\mathrm{CO}}$ $2062(\mathrm{~s}), 2021(\mathrm{~s}) \mathrm{cm}^{-1} ;{ }^{31} \mathrm{P}$ NMR (acetone- $\left.d_{6}\right): \delta 70.8(\mathrm{~s})$, -143.1 (hep, ${ }^{1} J_{\mathrm{PF}}=2829 \mathrm{~Hz}$ ); ${ }^{1} \mathrm{H}$ NMR (acetone- $\left.d_{6}\right): \delta$ 8.94 (b, 1H, Py), 7.46-7.96 (m, 13H, Ph and Py), 5.57 (b, $5 \mathrm{H}, \quad \mathrm{Cp}$ ); ${ }^{13} \mathrm{C}$ NMR (acetone- $d_{6}$ ): $\delta \quad 210.39$ (d, $\left.{ }^{2} J_{\mathrm{PC}}=25.67 \mathrm{~Hz}, \mathrm{CO}\right), 126.94-152.07$ (m, Ph and $\left.\mathrm{Py}\right)$, 89.28 (s, Cp); MS $(\mathrm{m} / z): 440\left(\mathbf{M}^{+}-\mathrm{PF}_{6}\right)$. Anal. Calc. for $\mathrm{C}_{24} \mathrm{H}_{19} \mathrm{NFeO}_{2} \mathrm{~F}_{6} \mathrm{P}_{2}: \mathrm{C}, 49.23 ; \mathrm{H}, 3.25 ; \mathrm{N}, 2.39$. Found: $\mathrm{C}$, 49.09; H, 3.14; N, 2.05\%.

\subsection{2. $\left[\left(\eta^{5}-\mathrm{C}_{5} \mathrm{H}_{5}\right) \mathrm{Fe}(\mathrm{CO})_{2} \mathrm{PPhPy}_{2}{ }^{+}\right]\left[\mathrm{PF}_{6}^{-}\right]$}

Yield: $0.94 \mathrm{~g}(90 \%)$; mp: $205-206^{\circ} \mathrm{C}$; IR $\left(\mathrm{CH}_{2} \mathrm{Cl}_{2}\right): v_{\mathrm{CO}}$ $2063(\mathrm{~s}), 2021(\mathrm{~s}) \mathrm{cm}^{-1} ;{ }^{31} \mathrm{P}$ NMR (acetone- $\left.d_{6}\right): \delta 74.2(\mathrm{~s})$, -143.1 (hep, ${ }^{1} J_{\mathrm{PF}}=2829 \mathrm{~Hz}$ ); ${ }^{1} \mathrm{H}$ NMR (acetone- $d_{6}$ ): $\delta$ $8.96\left(\mathrm{~d}, 2 \mathrm{H},{ }^{3} J_{\mathrm{PH}}=4.04 \mathrm{~Hz}, \mathrm{Py}\right), 7.42-7.99(\mathrm{~m}, 11 \mathrm{H}, \mathrm{Ph}$ and $\mathrm{Py}), 5.58(\mathrm{~b}, 5 \mathrm{H}, \mathrm{Cp}) ;{ }^{13} \mathrm{C}$ NMR (acetone- $\left.d_{6}\right): \delta$ $210.38\left(\mathrm{~d},{ }^{2} J_{\mathrm{PC}}=24.45 \mathrm{~Hz}, \mathrm{CO}\right), 127.00-155.98(\mathrm{~m}, \mathrm{Ph}$ and Py), 89.03 (s, Cp); MS (m/z): $442\left(\mathrm{M}^{+}-\mathrm{PF}_{6}\right)$. Anal. Calc. for $\mathrm{C}_{23} \mathrm{H}_{18} \mathrm{~N}_{2} \mathrm{FeO}_{2} \mathrm{~F}_{6} \mathrm{P}_{2}$ : C 47.10; H, 3.07; N, 4.78 . Found: C, 46.33; H, 2.84; N, 4.36\%.

\subsection{3. $\left[\left(\eta^{5}-\mathrm{C}_{5} \mathrm{H}_{5}\right) \mathrm{Fe}(\mathrm{CO})_{2} \mathrm{PPy}_{3}{ }^{+}\right]\left[\mathrm{PF}_{6}{ }^{-}\right]$}

Yield: $0.45 \mathrm{~g}(77 \%)$; mp: $200-202{ }^{\circ} \mathrm{C}$; IR $\left(\mathrm{CH}_{2} \mathrm{Cl}_{2}\right): v_{\mathrm{CO}}$ $2061(\mathrm{~s}), 2020(\mathrm{~s}) \mathrm{cm}^{-1} ;{ }^{31} \mathrm{P}$ NMR (acetone- $\left.d_{6}\right): \delta 76.1(\mathrm{~s})$, -143.1 (hep, ${ }^{1} J_{\mathrm{PF}}=2829 \mathrm{~Hz}$ ), ${ }^{1} \mathrm{H}$ NMR (acetone- $d_{6}$ ): $\delta$ 8.95 (b, 3H, Рy) 7.51-7.98 (m, 9H, Py), 5.55 (b, 5H, Cp); ${ }^{13} \mathrm{C}$ NMR (acetone- $\left.d_{6}\right): \delta 210.22\left(\mathrm{~d},{ }^{2} J_{\mathrm{PC}}=24.00 \mathrm{~Hz}\right.$, CO), 127.00-155.75 (m, Py), 88.67 (s, Cp); MS ( $/ \mathrm{z}^{\mathrm{z}}$ ): 443 $\left(\mathrm{M}^{+}-\mathrm{PF}_{6}+1\right)$. Anal. Calc. for $\mathrm{C}_{22} \mathrm{H}_{17} \mathrm{~N}_{3} \mathrm{FeO}_{2} \mathrm{~F}_{6} \mathrm{P}_{2}$ : C 44.97; H, 2.90; N, 7.16. Found: C, 44.73; H, 2.72; N, 6.84\%.

\subsection{Phosphine exchange under ETC conditions}

$\left[\left(\eta^{5}-\mathrm{C}_{5} \mathrm{H}_{5}\right) \mathrm{Fe}(\mathrm{CO})_{2} \mathrm{PPh}_{n} \mathrm{Py}_{3-n}{ }^{+}\right]\left[\mathrm{PF}_{6}^{-}\right](0.45 \mathrm{mmol})$ and $\mathrm{PPh}_{m} \mathrm{Py}_{3-m}(0.15 \mathrm{mmol} \times 3, m=0-3, m \neq n)$ were completely dissolved in $\mathrm{CH}_{3} \mathrm{CN}(1.5 \mathrm{~mL})$ and transferred to a $10 \mathrm{~mm}$ co-axial NMR tube that has an inner tube filled

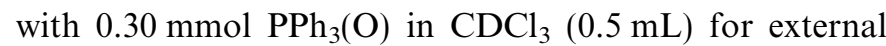
reference $\left(\delta=29.5\right.$ in ${ }^{31} \mathrm{P}$ NMR spectra) and for use in locking of the magnetic field. The 3:1:1:1 intensity integration ratio for the cation and the free ligands was observed from the ${ }^{31} \mathrm{P}$ NMR spectra. A trace amount of $\left(\eta^{5}\right.$ $\left.\mathrm{C}_{5} \mathrm{H}_{5}\right)_{2} \mathrm{Co}$ was then introduced to the solution in the NMR tube. The ${ }^{31} \mathrm{P}$ NMR spectrum was remeasured in which a total of eight peaks corresponding to $\left[\left(\eta^{5}-\mathrm{C}_{5} \mathrm{H}_{5}\right) \mathrm{Fe}(\mathrm{CO})_{2} \mathrm{PPh}_{m} \mathrm{Py}_{3-m}{ }^{+}\right]$and $\mathrm{PPh}_{n} \mathrm{Py}_{3-n} \quad(m$, $n=0-3)$ appeared. The intensity integration ratio was recorded again. The results are shown in Table 1.

\subsection{Preparation of $\left(\eta^{5}-\mathrm{C}_{5} \mathrm{H}_{5}\right) \mathrm{Fe}(\mathrm{CO}) \mathrm{C}(\mathrm{O}) \mathrm{Me}(\mathrm{L})$, $L=P P h_{3}, P P h_{2} P y, P P h P y_{2}, P P y_{3}$}

$\left(\eta^{5}-\mathrm{C}_{5} \mathrm{H}_{5}\right) \mathrm{Fe}(\mathrm{CO})_{2} \mathrm{Me}(0.50 \mathrm{~g}, 2.5 \mathrm{mmol})$ and the ligand $\mathrm{L}(2.5 \mathrm{mmol})$ were dissolved in THF $(10 \mathrm{~mL}$, freshly distilled) and refluxed for $48 \mathrm{~h}$. The mixture, an orange solution, was allowed to cool down to room temperature 
Table 1

The measured equilibrium constants of $[\mathrm{FpP} 1]^{+}+\mathrm{P} 2 \rightarrow \mathrm{P} 1+[\mathrm{FpP} 2]^{+\mathrm{a}}$

\begin{tabular}{|c|c|c|c|c|c|c|}
\hline Reactant & $\mathrm{PPh}_{3}$ & $\mathrm{PPh}_{2} \mathrm{Py}$ & $\mathrm{PPhPy}_{2}$ & $\mathrm{PPy}_{3}$ & & \\
\hline $\mathrm{FpPPh}_{3}{ }^{+}$ & $\begin{array}{l}1 \\
1 \\
1 \\
1 \\
K_{11}=1\end{array}$ & $\begin{array}{l}4.76^{\mathrm{b}} \\
5.41^{\mathrm{c}} \\
5.05^{\mathrm{d}} \\
4.36^{\mathrm{e}} \\
K_{12}=4.90(44)^{\mathrm{f}}\end{array}$ & $\begin{array}{l}10.7 \\
11.4 \\
11.2 \\
10.7 \\
K_{13}=11.0(4)\end{array}$ & $\begin{array}{l}18.7 \\
25.6 \\
20.4 \\
16.7 \\
K_{14}=20.3(38)\end{array}$ & $\mathrm{PPh}_{3}$ & \\
\hline $\mathrm{FpPPh}_{2} \mathrm{Py}^{+}$ & $\begin{array}{l}0.210 \\
0.185 \\
0.198 \\
0.229 \\
K_{21}=0.205(19)\end{array}$ & $\begin{array}{l}1 \\
1 \\
1 \\
1 \\
K_{22}=1\end{array}$ & $\begin{array}{l}2.25 \\
2.11 \\
2.22 \\
2.45 \\
K_{23}=2.25(14)\end{array}$ & $\begin{array}{l}3.93 \\
4.74 \\
4.03 \\
3.69 \\
K_{24}=4.10(45)\end{array}$ & $\mathrm{PPh}_{2} \mathrm{Py}$ & \\
\hline $\mathrm{FpPPhPy}_{2}{ }^{+}$ & $\begin{array}{l}0.0932 \\
0.0876 \\
0.0893 \\
0.0938 \\
K_{31}=0.0910(30)\end{array}$ & $\begin{array}{l}0.444 \\
0.474 \\
0.450 \\
0.408 \\
K_{32}=0.444(27)\end{array}$ & $\begin{array}{l}1 \\
1 \\
1 \\
1 \\
K_{33}=1\end{array}$ & $\begin{array}{l}1.74 \\
2.24 \\
1.82 \\
1.51 \\
K_{34}=1.83(31)\end{array}$ & $\mathrm{PPhPy}_{2}$ & \\
\hline $\mathrm{FpPPy}_{3}{ }^{+}$ & $\begin{array}{l}0.0535 \\
0.0390 \\
0.0491 \\
0.0599 \\
K_{14}=0.0504(88)\end{array}$ & $\begin{array}{l}0.254 \\
0.211 \\
0.248 \\
0.271 \\
K_{42}=0.246(25)\end{array}$ & $\begin{array}{l}0.575 \\
0.446 \\
0.549 \\
0.662 \\
K_{43}=0.558(89)\end{array}$ & $\begin{array}{l}1 \\
1 \\
1 \\
1 \\
K_{44}=1\end{array}$ & $\mathrm{PPy}_{3}$ & \\
\hline & $\mathrm{FpPPh}_{3}{ }^{+}$ & $\mathrm{FpPPh}_{2} \mathrm{Py}^{+}$ & & $\mathrm{FpPPhPy}_{2}{ }^{+}$ & $\mathrm{FpPPy}_{3}{ }^{+}$ & Product \\
\hline
\end{tabular}

${ }^{\mathrm{a}} \mathrm{Fp}=\left(\eta^{5}-\mathrm{C}_{5} \mathrm{H}_{5}\right) \mathrm{Fe}(\mathrm{CO})_{2}, \mathrm{P} 1, \mathrm{P} 2=\mathrm{PPh}_{3}, \mathrm{PPh}_{2} \mathrm{Py}, \mathrm{PPhPy}_{2}, \mathrm{PPy}_{3}$.

${ }^{b}$ With the initial condition: $3 \mathrm{FpPPh}_{3}{ }^{+} \mathrm{PF}_{6}{ }^{-}+\mathrm{PPh}_{2} \mathrm{Py}+\mathrm{PPhPy}_{2}+\mathrm{PPy}_{3}$.

${ }^{\mathrm{c}}$ With the initial condition: $3 \mathrm{FpPPh}_{2} \mathrm{Py}^{+} \mathrm{PF}_{6}{ }^{-}+\mathrm{PPh}_{3}+\mathrm{PPhPy}_{2}+\mathrm{PPy}_{3}$.

${ }^{\mathrm{d}}$ With the initial condition: $3 \mathrm{FpPPhPy}_{2}{ }^{+} \mathrm{PF}_{6}{ }^{-}+\mathrm{PPh}_{3}+\mathrm{PPh}_{2} \mathrm{Py}+\mathrm{PPy}_{3}$.

${ }^{\mathrm{e}}$ With the initial condition: $3 \mathrm{FpPPy}_{3}{ }^{+} \mathrm{PF}_{6}{ }^{-}+\mathrm{PPh}_{3}+\mathrm{PPh}_{2} \mathrm{Py}+\mathrm{PPhPy}_{2}$.

${ }^{\mathrm{f}}$ Average (standard deviation) of experiments b-e.

then the insoluble solid was removed by passing through a pad of celite. The solvent was removed under vacuum. The residue was dissolved in minimal $\mathrm{CH}_{2} \mathrm{Cl}_{2}$, mixed well with a small quantity of alumina and packed on the top of an alumina column. The bands were eluted first with 1:10 EtOAc/hexane to remove the trace amount of yellow $\left(\eta^{5}-\right.$ $\left.\mathrm{C}_{5} \mathrm{H}_{5}\right) \mathrm{Fe}(\mathrm{CO})_{2} \mathrm{Me}$. The acetyl complexes $\left(\eta^{5}-\mathrm{C}_{5} \mathrm{H}_{5}\right) \mathrm{Fe}$ $(\mathrm{CO}) \mathrm{C}(\mathrm{O}) \mathrm{Me}(\mathrm{L})$ were collected using eluents of higher polarity: with 1:5 EtOAc/hexane for $\mathrm{L}=\mathrm{PPh}_{3}$; with 1:2 EtOAc/hexane for $\mathrm{L}=\mathrm{PPh}_{2} \mathrm{Py}$; with $3: 1 \mathrm{EtOAc} /$ hexane for $\mathrm{L}=\mathrm{PPhPy}_{2}$; and with 3.5:1 EtOAc/hexane for $\mathrm{L}=\mathrm{PPy}_{3}$.

$\left(\eta^{5}-\mathrm{C}_{5} \mathrm{H}_{5}\right) \mathrm{Fe}(\mathrm{CO}) \mathrm{C}(\mathrm{O}) \mathrm{Me}\left(\mathrm{PPh}_{3}\right)$ : yield $0.59 \mathrm{~g}(59 \%)$; IR $\left(\mathrm{CH}_{2} \mathrm{Cl}_{2}\right): v_{\mathrm{CO}} 1916(\mathrm{~s}) 1601(\mathrm{~m}) \mathrm{cm}^{-1} ;{ }^{31} \mathrm{P} \mathrm{NMR}\left(\mathrm{CDCl}_{3}\right)$ : $\delta 75.23$ (s); Literature [27] IR $\left(\mathrm{CHCl}_{3}\right): v_{\mathrm{CO}} 1920$ (vs, br), $1598(\mathrm{~s}) \mathrm{cm}^{-1}$.

$\left(\eta^{5}-\mathrm{C}_{5} \mathrm{H}_{5}\right) \mathrm{Fe}(\mathrm{CO}) \mathrm{C}(\mathrm{O}) \mathrm{Me}\left(\mathrm{PPh}_{2} \mathrm{Py}\right)$ : yield $0.53 \mathrm{~g}(47 \%)$; mp: $132-133{ }^{\circ} \mathrm{C}$; IR $\left(\mathrm{CH}_{2} \mathrm{Cl}_{2}\right): v_{\mathrm{CO}} 1921$ (s), 1599 (m) $\mathrm{cm}^{-1} ;{ }^{31} \mathrm{P}$ NMR $\left(\mathrm{CDCl}_{3}\right): \delta 80.9(\mathrm{~s}) ;{ }^{1} \mathrm{H}$ NMR $\left(\mathrm{CDCl}_{3}\right)$ : $\delta 8.68\left(\mathrm{~d},{ }^{4} J_{\mathrm{PH}}=3.94 \mathrm{~Hz}, 1 \mathrm{H}, \mathrm{Py}\right), 7.18-7.60(\mathrm{~m}, 13 \mathrm{H}$, $\mathrm{Ph}$ and $\mathrm{Py}), 4.44$ (s, 5H Cp), 2.38(s, 3H, Me); ${ }^{13} \mathrm{C}$ NMR $\left(\mathrm{CDCl}_{3}\right): \delta 276.83(\mathrm{~b}, \mathrm{C}(\mathrm{O}) \mathrm{Me}), 220.07$ (b, CO), 123.13$161.14(\mathrm{~m}, \mathrm{Ph}$ and $\mathrm{Py}), 84.96$ (s, Cp), 52.18 (s, Me); MS $(\mathrm{m} / z): 466\left(\mathrm{M}^{+}+1\right)$. Anal. Calc. for $\mathrm{C}_{25} \mathrm{H}_{22} \mathrm{NO}_{2} \mathrm{FeP}: \mathrm{C}$, 65.93; H, 4.84; N, 3.08. Found: C, 65.83; H, 4.94; N, 2.87\%.

$\left(\eta^{5}-\mathrm{C}_{5} \mathrm{H}_{5}\right) \mathrm{Fe}(\mathrm{CO}) \mathrm{C}(\mathrm{O}) \mathrm{Me}\left(\mathrm{PPhPy}_{2}\right)$ : yield $0.55 \mathrm{~g}(48 \%)$; mp: $136-137^{\circ} \mathrm{C}$; IR $\left(\mathrm{CH}_{2} \mathrm{Cl}_{2}\right): v_{\mathrm{CO}} 1923$ (s), 1598 (m) $\mathrm{cm}^{-1} ;{ }^{31} \mathrm{P}$ NMR $\left(\mathrm{CDCl}_{3}\right): \delta 84.6(\mathrm{~s}) ;{ }^{1} \mathrm{H}$ NMR $\left(\mathrm{CDCl}_{3}\right)$ : $\delta 8.66(\mathrm{~b}, 2 \mathrm{H}, \mathrm{Py}), 7.20-7.62(\mathrm{~m}, 12 \mathrm{H}, \mathrm{Ph}$ and $\mathrm{Py}), 4.48$ $(\mathrm{s}, 5 \mathrm{H}, \mathrm{Cp}), 2.41(\mathrm{~s}, 3 \mathrm{H}, \mathrm{Me}) ;{ }^{13} \mathrm{C} \mathrm{NMR}\left(\mathrm{CDCl}_{3}\right): \delta$ $276.01 \quad\left(\mathrm{~d}, \quad{ }^{3} J_{\mathrm{PC}}=22 \mathrm{~Hz}, \quad \mathrm{C}(\mathrm{O}) \mathrm{Me}\right), \quad 219.92 \quad(\mathrm{~d}$, $\left.{ }^{3} J_{\mathrm{PC}}=30 \mathrm{~Hz}, \mathrm{CO}\right), 123.14-161.00(\mathrm{~m}, \mathrm{Ph}$ and $\mathrm{Py}), 84.34$ (s, Cp), 51.90 (s, Me); MS (m/z): $457\left(\mathrm{M}^{+}+1\right)$; Anal. Calc. for $\mathrm{C}_{24} \mathrm{H}_{21} \mathrm{~N}_{2} \mathrm{PFeO}_{2}$ : C, 63.16; $\mathrm{H}, 4.61 ; \mathrm{N}, 6.14$. Found: $\mathrm{C}$, 63.51; H, 4.70; N, 5.99\%.

$\left(\eta^{5}-\mathrm{C}_{5} \mathrm{H}_{5}\right) \mathrm{Fe}(\mathrm{CO}) \mathrm{C}(\mathrm{O}) \mathrm{Me}\left(\mathrm{PPy}_{3}\right)$ : yield $0.58 \mathrm{~g} \mathrm{(51 \% );}$ mp: $127-128{ }^{\circ} \mathrm{C}$; IR $\left(\mathrm{CH}_{2} \mathrm{Cl}_{2}\right): v_{\mathrm{CO}} 1927$ (s) 1597 (m) $\mathrm{cm}^{-1} ;{ }^{31} \mathrm{P}$ NMR $\left(\mathrm{CDCl}_{3}\right): \delta 89.7(\mathrm{~s}) ;{ }^{1} \mathrm{H}$ NMR $\left(\mathrm{CDCl}_{3}\right)$ : $\delta 8.64$ (b, 3H, Py), 7.84 (b, 3H, Py), 7.66 (b, 3H, Py), 7.22 (b, 3H, Py), 4.51 (s, 5H, Cp), 2.43 (s, 3H, Me); ${ }^{13} \mathrm{C}$ NMR $\left(\mathrm{CDCl}_{3}\right): \delta 275.93\left(\mathrm{~d},{ }^{3} J_{\mathrm{PC}}=23 \mathrm{~Hz}, \mathrm{C}(\mathrm{O}) \mathrm{Me}\right)$, $219.70\left(\mathrm{~d},{ }^{3} J_{\mathrm{PC}}=30 \mathrm{~Hz}, \mathrm{CO}\right), 122.76-161.81(\mathrm{~m}, \mathrm{Ph}$ and Py), $84.62(\mathrm{~s}, \mathrm{Cp}), 51.81\left(\mathrm{~d}, \mathrm{Me},{ }^{3} J_{\mathrm{PC}}=4 \mathrm{~Hz}\right) ; \mathrm{MS}(\mathrm{m} / \mathrm{z})$ : $458\left(\mathrm{M}^{+}+1\right)$; Anal. Calc. for $\mathrm{C}_{23} \mathrm{H}_{20} \mathrm{~N}_{3} \mathrm{PFeO}_{2}$ : C, 60.39; $\mathrm{H}, 4.38$; N, 9.19. Found: C, 60.23, H4.41; N, 9.36\%.

\subsection{Cyclic voltammetry study}

The cyclic voltammograms of $10^{-3} \mathrm{M}\left[\left(\eta^{5}-\mathrm{C}_{5} \mathrm{H}_{5}\right) \mathrm{Fe}-\right.$ $\left.(\mathrm{CO})_{2} \mathrm{~L}^{+}\right]\left[\mathrm{PF}_{6}{ }^{-}\right]$and $\left(\eta^{5}-\mathrm{C}_{5} \mathrm{H}_{5}\right) \mathrm{Fe}(\mathrm{CO}) \mathrm{C}(\mathrm{O}) \mathrm{Me}(\mathrm{L})$ where $\mathrm{L}=\mathrm{PPh}_{3}, \mathrm{PPh}_{2} \mathrm{Py}, \mathrm{PPhPy}_{2}$, and $\mathrm{PPy}_{3}$ were measured at room temperature on $10^{-1} \mathrm{M}\left[\mathrm{Bu}_{4} \mathrm{~N}^{+}\right]\left[\mathrm{PF}_{6}{ }^{-}\right]$in $\mathrm{CH}_{2} \mathrm{Cl}_{2}$ solution. The working electrode was a glassy cabon electrode. The reference electrode was $\mathrm{Ag} / \mathrm{AgNO}_{3}$ for use in non-aqueous systems. The scan rate was $100 \mathrm{mV} / \mathrm{s}$ in the range of -1500 to $0 \mathrm{mV}$ for $\left[\left(\eta^{5}-\mathrm{C}_{5} \mathrm{H}_{5}\right) \mathrm{Fe}(\mathrm{CO})_{2} \mathrm{~L}^{+}\right]$ 
complexes and in the range of -500 to $+500 \mathrm{mV} / \mathrm{s}$ for acetyl complexes, potentials being relative to ferrocene $e^{0,+}$ $(0.281 \mathrm{~V})$. For acetyl complexes with the phosphines bearing at least one Py group, only oxidative waves were recorded, no corresponding reductive peaks being observed. For $\left[\left(\eta^{5}-\mathrm{C}_{5} \mathrm{H}_{5}\right) \mathrm{Fe}(\mathrm{CO})_{2} \mathrm{~L}^{+}\right]$complexes, only reductive and no oxidative waves were observed. The following is the listing of measured potentials:

\begin{tabular}{lc}
\hline Complex & Potential (V) \\
\hline$\left(\eta^{5}-\mathrm{C}_{5} \mathrm{H}_{5}\right)_{2} \mathrm{Fe}{ }^{0,+}$ & 0.281 \\
$\left(\eta^{5}-\mathrm{C}_{5} \mathrm{H}_{5}\right) \mathrm{Fe}(\mathrm{CO}) \mathrm{C}(\mathrm{O}) \mathrm{Me}\left(\mathrm{PPh}_{3}\right)^{0,+}$ & 0.244 \\
$\left(\eta^{5}-\mathrm{C}_{5} \mathrm{H}_{5}\right) \mathrm{Fe}(\mathrm{CO}) \mathrm{C}(\mathrm{O}) \mathrm{Me}\left(\mathrm{PPh}_{2} \mathrm{Py}\right)^{0,+}$ & $0.227^{\mathrm{a}}$ \\
$\left(\eta^{5}-\mathrm{C}_{5} \mathrm{H}_{5}\right) \mathrm{Fe}(\mathrm{CO}) \mathrm{C}(\mathrm{O}) \mathrm{Me}\left(\mathrm{PPhPy}_{2}\right)^{0,+}$ & $0.200^{\mathrm{a}}$ \\
$\left(\eta^{5}-\mathrm{C}_{5} \mathrm{H}_{5}\right) \mathrm{Fe}(\mathrm{CO}) \mathrm{C}(\mathrm{O}) \mathrm{Me}\left(\mathrm{PPy}_{3}\right)^{0,+}$ & $0.195^{\mathrm{a}}$ \\
$\left(\eta^{5}-\mathrm{C}_{5} \mathrm{H}_{5}\right) \mathrm{Fe}(\mathrm{CO})_{2} \mathrm{PPh}_{3}^{+, 0}$ & $-1.234^{\mathrm{b}}$ \\
$\left(\eta^{5}-\mathrm{C}_{5} \mathrm{H}_{5}\right) \mathrm{Fe}(\mathrm{CO})_{2} \mathrm{PPh}_{2} \mathrm{Py}^{+, 0}$ & $-1.353^{\mathrm{b}}$ \\
$\left(\eta^{5}-\mathrm{C}_{5} \mathrm{H}_{5}\right) \mathrm{Fe}(\mathrm{CO})_{2} \mathrm{PPhPy}_{2}^{+, 0}$ & $-1.380^{\mathrm{b}}$ \\
$\left(\eta^{5}-\mathrm{C}_{5} \mathrm{H}_{5}\right) \mathrm{Fe}(\mathrm{CO})_{2} \mathrm{PPy}_{3}{ }^{+, 0}$ & $-1.414^{\mathrm{b}}$ \\
\hline
\end{tabular}

${ }^{\mathrm{a}}$ Only oxidative wave observed.

${ }^{\mathrm{b}}$ Only reductive wave observed.

\section{Results and discussion}

The preparation of $\left(\eta^{5}-\mathrm{C}_{5} \mathrm{H}_{5}\right) \mathrm{Fe}(\mathrm{CO}) \mathrm{C}(\mathrm{O}) \mathrm{MePPh}_{n} \mathrm{Py}_{3-n}$ complexes proceeded smoothly, following the literature procedure of $\left(\eta^{5}-\mathrm{C}_{5} \mathrm{H}_{5}\right) \mathrm{Fe}(\mathrm{CO}) \mathrm{C}(\mathrm{O}) \mathrm{MePPh}_{3}$ [27] and gave $47-59 \%$ isolated yields. The $\mathrm{PPh}_{n} \mathrm{Py}_{3-n}$ analogs were characterized with $\mathrm{mp}$, IR, NMR, MS, and elemental analysis, where the spectroscopic data are reasonable. The $v_{\mathrm{CO}}$ stretching frequencies of the acetyl complexes increase in the following order, e.g., $1916 \mathrm{~cm}^{-1}$ for the $\mathrm{PPh}_{3}$ complex, $1921 \mathrm{~cm}^{-1}$ for the $\mathrm{PPh}_{2} \mathrm{Py}$ complex, $1923 \mathrm{~cm}^{-1}$ for the $\mathrm{PPhPy}_{2}$ complex, and $1927 \mathrm{~cm}^{-1}$ for the $\mathrm{PPy}_{3}$ complex. Clearly there is a decrease in backbonding from $\mathrm{Fe}$ to the CO ligand with an increase of Py-groups, suggesting that $\mathrm{PPy}_{3}$ is the least donating arylpyridylphosphine in the series. On the other hand, the CV oxidative wave of the acetyl complex shows a decrease in potential $\left(0.244 \mathrm{~V}, 0.227_{\text {irrev }} \mathrm{V}\right.$, $0.200_{\text {irrev }} \mathrm{V}$, and $0.195_{\text {rrev }} \mathrm{V}$ corresponding to $0,1,2$, and 3 Py groups), indicative of $\mathrm{PPy}_{3}$ the best donating ligand in the series, making $\left(\eta^{5}-\mathrm{C}_{5} \mathrm{H}_{5}\right) \mathrm{Fe}(\mathrm{CO}) \mathrm{C}(\mathrm{O}) \mathrm{MePPy}_{3}$, the easiest to be oxidized. The $v_{\mathrm{CO}}$ stretching frequencies and $E^{\circ}$ values are in opposite directions! The $\mathrm{pK}$ value of $\mathrm{HPPh}_{n} \mathrm{Py}_{3-n}+$ is not a good indicator here because of pyridyl-N complication to the $\mathrm{P}$ base site - the titration curves of $\mathrm{PPh}_{n} \mathrm{Py}_{3-n}(n=1-3)$ using an acid are hardly resolved. It is not conclusive, based on one of the Giering's parameters, to specify the best donor ligand in the $\mathrm{PPh}_{n} \mathrm{Py}_{3-n}$ series.

The preparation of $\left.\left(\eta^{5}-\mathrm{C}_{5} \mathrm{H}_{5}\right) \mathrm{Fe}(\mathrm{CO})_{2} \mathrm{PPh}_{n} \mathrm{Py}_{3-n}{ }^{+}\right]$ $\left[\mathrm{PF}_{6}{ }^{+}\right]$follows the ETC $\mathrm{PPh}_{n} \mathrm{Py}_{3-n}$ ligand replacement for $\mathrm{I}^{-}$on $\left(\eta^{5}-\mathrm{C}_{5} \mathrm{H}_{5}\right) \mathrm{Fe}(\mathrm{CO})_{2} \mathrm{I}$. The yields are excellent (in $77-90 \%$ isolated yields) and the spectroscopic data are in agreement with the structural formula.
The ${ }^{31} \mathrm{P}$ resonances of free $\mathrm{PPh}_{n} \mathrm{Py}_{3-n}$ ligands are seen to be more downfield with more Py groups $(\delta-4.7,-3.2$, -1.9 , and -0.6 for $0,1,2$ and 3 Py-groups, respectively). Given a Ph-group contribution as $\sigma_{\mathrm{Ph}}$ and Py-group contribution as $\sigma_{\mathrm{Py}}$, the relationship between the chemical shifts and the group contributions is linear, namely,

$\delta($ free ligand $)=-4.65 \delta+\sum_{i} \sigma_{i}$

where $\sigma_{i}$ is $\sigma_{\mathrm{Ph}}(=0.00 \delta)$ and $\sigma_{\mathrm{py}}(=1.36 \delta)$. Upon complexation, the ${ }^{31} \mathrm{P}$ resonances of the acetyl complexes $\left(\eta^{5}\right.$ $\left.\mathrm{C}_{5} \mathrm{H}_{5}\right) \mathrm{Fe}(\mathrm{CO}) \mathrm{C}(\mathrm{O}) \mathrm{MePPh}_{n} \mathrm{Py}_{3-n}$ are $\delta$ 75.2, 80.9, 84.6, and 89.7, respectively, in the order of increasing number of Py-groups. The complexation shifts $\Delta(=\delta$ of complex $-\delta$ of ligand) are 79.9-90.3 $\delta$ downfield. Employing the same group contribution $\sigma_{i}$ as for the free ligands, the ${ }^{31} \mathrm{P}$ chemical shifts of the acetyl complexes could be calculated within $\pm 0.7 \delta$, in the linear fitting as follows:

$\delta_{(\text {acetyl complex })}=75.52 \delta+3.47\left(\sum_{i} \sigma_{i}\right)$

where a ratio of 3.47 is necessary. Greater than 1.00 , this constant suggests an amplification about the phosphine group contributions upon complexation. The similar analysis, when applied to the $\left[\left(\eta^{5}-\mathrm{C}_{5} \mathrm{H}_{5}\right) \mathrm{Fe}(\mathrm{CO})_{2} \mathrm{PPh}_{n} \mathrm{Py}_{3-n}{ }^{+}\right]$ series, results in complexation shifts $\Delta$ ranging from 67.2 to $76.6 \delta$ downfield, and a linear fitting of

$\delta_{\text {(cation complex) }}=64.13 \delta+3.25\left(\sum_{i} \sigma_{i}\right)$.

The calculated chemical shifts and the observed ones are within $\pm 2.0 \delta$. The ratio 3.25 is also suggestive of an amplification. Upon complexation, the $\mathrm{PPh}_{n} \mathrm{Py}_{3-n}$ series is apparently different from the $\mathrm{PPh}_{n} \mathrm{Me}_{3-n}$ series because in the latter case, the linear regression for ligands $\mathrm{PPh}_{n} \mathrm{Me}_{3-n}$ and the corresponding complexes $\left(\eta^{4}\right.$-exo$\left.\mathrm{MeC}_{5} \mathrm{H}_{5}\right) \mathrm{Fe}(\mathrm{CO})_{2} \mathrm{PPh}_{n} \mathrm{Me}_{3-n}$ indicates a regulation, instead of an amplification, about the phosphine group contributions upon complexation where the ratio is only 0.85 that is less than 1.00 [28].

The $v_{\mathrm{CO}}$ stretching frequencies of the $\left[\left(\eta^{5}-\mathrm{C}_{5} \mathrm{H}_{5}\right)\right.$ $\left.\mathrm{Fe}(\mathrm{CO})_{2} \mathrm{PPh}_{n} \mathrm{Py}_{3-n}^{+}\right]$series are not very much different and are not conclusive in differentiating the $\mathrm{PPh}_{n} \mathrm{Py}_{3-n}$ donor capability at all. Similar to the reported $\mathrm{CV}$ of $\left[\left(\eta^{5}-\mathrm{C}_{5} \mathrm{H}_{5}\right) \mathrm{Fe}(\mathrm{CO})_{2} \mathrm{PPh}_{3}{ }^{+}\right]$by Stweigart et al. [18], the cyclic voltammograms of $\left[\left(\eta^{5}-\mathrm{C}_{5} \mathrm{H}_{5}\right) \mathrm{Fe}(\mathrm{CO})_{2} \mathrm{PPh}_{n} \mathrm{Py}_{3-n}{ }^{+}\right]$in our study gave irreversible, reductive waves $(-1.234 \mathrm{~V}$, $-1.353 \mathrm{~V},-1.380 \mathrm{~V},-1.414 \mathrm{~V}$, in the order of $0,1,2$, and 3 Py groups), indicative of a reactivity after being reduced. It is then worth trying the phosphine-exchange experiments. A $10 \mathrm{~mm}$ coaxial NMR tube has been employed that has an inner-tube containing $\mathrm{PPh}_{3}(\mathrm{O}) / \mathrm{CDCl}_{3}$ and the outer-tube containing a $\mathrm{CH}_{3} \mathrm{CN}$ solution of the $\left(\eta^{5}-\mathrm{C}_{5} \mathrm{H}_{5}\right) \mathrm{Fe}(\mathrm{CO})_{2}$ complex and phosphines. For example, the ${ }^{31} \mathrm{P}$ NMR spectrum for a $3: 1: 1: 1$ mixture of $\left[\left(\eta^{5}-\mathrm{C}_{5} \mathrm{H}_{5}\right) \mathrm{Fe}(\mathrm{CO})_{2} \mathrm{PPh}_{3}{ }^{+}\right] /$ $\mathrm{PPy}_{3} / \mathrm{PPhPy}_{2} / \mathrm{PPh}_{2} \mathrm{Py}$ gave respective peaks at $\delta 62.5$, 
$-0.6,-1.9,-3.2$ and intensity integration ratios of ca. 19:6:6:6. After the addition of trace amount of $\left(\eta^{5}\right.$ $\left.\mathrm{C}_{5} \mathrm{H}_{5}\right)_{2} \mathrm{Co}$, an initiator to activate the $17 \mathrm{e}-19 \mathrm{e}$ pathway, the mixture reveals a ${ }^{31} \mathrm{P}$ NMR spectrum of $\mathrm{FpPPy}_{3}{ }^{+}$, $\mathrm{FpPPhPy}_{2}{ }^{+}, \mathrm{FpPPh}_{2} \mathrm{Py}^{+}, \mathrm{FpPPh}_{3}{ }^{+}, \mathrm{PPy}_{3}, \mathrm{PPhPy}_{2}, \mathrm{PPh}_{2} \mathrm{Py}$, $\mathrm{PPh}_{3}$ with respective peaks at $\delta 76.1,74.0,70.5,62.5,-0.6$, $-1.9,-3.2,-4.7$ and intensity integration ratios of ca. 10.80:10.47:8.08:7.94:2.36:3.99:6.94:32.46, respectively. The change of the spectrum is both instantaneous and extensive. The time delay between two spectra is less than a few minutes, barely enough for one to introduce the $\left(\eta^{5}-\mathrm{C}_{5} \mathrm{H}_{5}\right)_{2} \mathrm{Co}$ reductant to the $10 \mathrm{~mm}$ NMR tube and set up a new NMR experiment. Four new species are formed after addition of $\left(\eta^{5}-\mathrm{C}_{5} \mathrm{H}_{5}\right)_{2} \mathrm{Co}-$ the peak at $\delta-4.7$ is indicative of released $\mathrm{PPh}_{3}$ and the three new resonances at $\delta$ 76.1, 74.0, and 70.5 gave evidence of the formation of $\left[\left(\eta^{5}-\mathrm{C}_{5} \mathrm{H}_{5}\right) \mathrm{Fe}(\mathrm{CO})_{2} \mathrm{PPy}_{3}{ }^{+}\right], \quad\left[\left(\eta^{5}-C_{5} \mathrm{H}_{5}\right) \mathrm{Fe}(\mathrm{CO})_{2} \mathrm{PPhPy}_{2}{ }^{+}\right]$, and $\left[\left(\eta^{5}-\mathrm{C}_{5} \mathrm{H}_{5}\right) \mathrm{Fe}(\mathrm{CO})_{2} \mathrm{PPh}_{2} \mathrm{Py}^{+}\right]$, respectively. The intensity integration ratios for $\left[\left(\eta^{5}-\mathrm{C}_{5} \mathrm{H}_{5}\right) \mathrm{Fe}(\mathrm{CO})_{2} \mathrm{PPy}_{3}{ }^{+}\right]$, $\left[\left(\eta^{5}-\mathrm{C}_{5} \mathrm{H}_{5}\right) \mathrm{Fe}(\mathrm{CO})_{2} \mathrm{PPhPy}_{2}{ }^{+}\right], \quad\left[\left(\eta^{5}-\mathrm{C}_{5} \mathrm{H}_{5}\right) \mathrm{Fe}(\mathrm{CO})_{2} \mathrm{PPh}_{2^{-}}\right.$ $\left.\mathrm{Py}^{+}\right],\left[\left(\eta^{5}-\mathrm{C}_{5} \mathrm{H}_{5}\right) \mathrm{Fe}(\mathrm{CO})_{2} \mathrm{PPh}_{3}{ }^{+}\right], \mathrm{PPy}_{3}, \mathrm{PhPy}_{2}, \mathrm{PPh}_{2} \mathrm{Py}$, and $\mathrm{PPh}_{3}$ (in decreasing order of $\delta$ values) become ca. $11: 10: 8: 8: 2: 4: 7: 32$. The ratios stay the same after $24 \mathrm{~h}$, i.e., the phosphine exchange reaction with a radical process proceeds to a completion in a time scale of sub-minutes.

For a relevant pair of phosphines, the equilibrium constant $K$ of Eq. (1) could be calculated based on the intensity integration ratio. Thus, out of 4 complexes and 4 ligands involved after the addition of $\left(\eta^{5}-\mathrm{C}_{5} \mathrm{H}_{5}\right)_{2} \mathrm{Co}$, there are 16 phosphine exchange equilibrium constants $K_{i j}(i, j=1-4)$, defined in Table 1, among which four are the self-exchange $\left(K_{11}=K_{22}=K_{33}=K_{44}=1\right)$, six the forward reactions $\left(K_{i j}\right.$ with $i<j)$ and six the reverse reactions $\left(K_{i j}\right.$ with $\left.i>j\right)$. For instance in the equation

$$
\begin{aligned}
&\left(\eta^{5}-\mathrm{C}_{5} \mathrm{H}_{5}\right) \mathrm{Fe}(\mathrm{CO})_{2} \mathrm{PPh}_{3}{ }^{+}+\mathrm{PPy}_{3} \\
&=\mathrm{PPh}_{3}+\left(\eta^{5}-\mathrm{C}_{5} \mathrm{H}_{5}\right) \mathrm{Fe}(\mathrm{CO})_{2} \mathrm{PPy}_{3}{ }^{+} \\
& K_{14}=\left[\left(\eta^{5}-\mathrm{C}_{5} \mathrm{H}_{5}\right) \mathrm{Fe}(\mathrm{CO})_{2} \mathrm{PPy}_{3}{ }^{+}\right]\left[\mathrm{PPh}_{3}\right] \\
& \\
& \quad /\left[\left(\eta^{5}-\mathrm{C}_{5} \mathrm{H}_{5}\right) \mathrm{Fe}(\mathrm{CO})_{2} \mathrm{PPh}_{3}{ }^{+}\right]\left[\mathrm{PPy}_{3}\right] \\
& \equiv(11)(32) /(8)(2)=22 .
\end{aligned}
$$

Listed in Table 1, the actual calculations have been performed using the intensity integration values in two decimal points ( $K_{14}$ is 18.7 , instead of the approximate 22 ). The rest of equilibrium constants are also calculated in this manner. The conversion factors relating the concentration of a $\mathrm{PPh}_{n} \mathrm{Py}_{3-n}$ or a $\left[\left(\eta^{5}-\mathrm{C}_{5} \mathrm{H}_{5}\right) \mathrm{Fe}(\mathrm{CO})_{2} \mathrm{PPh}_{n} \mathrm{Py}_{3-n}{ }^{+}\right]$and the intensity integration in ${ }^{31} \mathrm{P}$ NMR spectrum may be different. In our experience, these factors are about the same if an error of $\pm 10 \%$ is taken into consideration. Experiments with simultaneously present arylpyridylphosphines indeed deliver a very nice internal consistency. The initial concentrations with three portions of one $\left(\eta^{5}-\mathrm{C}_{5} \mathrm{H}_{5}\right)$ $\mathrm{Fe}(\mathrm{CO})_{2}{ }^{-}$phosphine complex and one portion each of the remaining arylpyridylphosphines are listed in Table 1 that summarizes the phosphine exchange results.

\section{1. $\mathrm{Fp}^{+}$-affinity for $P \mathrm{Ph}_{n} P y_{3-n}$}

The equilibrium constants in Table 1 clearly reveal that for the series of arylpyridylphosphines $\mathrm{PPh}_{n} \mathrm{Py}_{3-n}$, the donor capability increases in the order $\mathrm{PPh}_{3}<\mathrm{PPh}_{2} \mathrm{Py}$ $<\mathrm{PPhPy}_{2}<\mathrm{PPy}_{3}$. The standard deviations of $K_{i j}$ 's are within $20 \%$ which seems reasonable in view of uncorrected instrumentation factors. These equilibrium constants could further be deducted into a much more easily understood $\mathrm{Fp}^{+}$-affinity scale where $\mathrm{Fp}^{+}=\left(\eta^{5}-\mathrm{C}_{5} \mathrm{H}_{5}\right) \mathrm{Fe}(\mathrm{CO})_{2}{ }^{+}$, which is a measure of the association between a $\mathrm{PPh}_{n} \mathrm{Py}_{3-n}$ ligand and the $\mathrm{Fp}^{+}$cation:

$$
\begin{array}{ll}
\mathrm{Fp}^{+}+\mathrm{PPh}_{3}=\mathrm{FpPPh}_{3}{ }^{+} & K_{1}=\left[\mathrm{FpPPh}_{3}^{+}\right] /\left[\mathrm{Fp}^{+}\right]\left[\mathrm{PPh}_{3}\right] \\
\mathrm{Fp}^{+}+\mathrm{PPh}_{2} \mathrm{Py}=\mathrm{FpPPh}_{2} \mathrm{Py}^{+} & K_{2}=\left[\mathrm{FpPPh}_{2} \mathrm{Py}^{+}\right] /\left[\mathrm{Fp}^{+}\right]\left[\mathrm{PPh}_{2} \mathrm{Py}^{2}\right] \\
\mathrm{Fp}^{+}+\mathrm{PPhPy}_{2}=\mathrm{FpPPhPy}_{2}{ }^{+} K_{3}=\left[\mathrm{FpPPPy}_{2}^{+}\right] /\left[\mathrm{Fp}^{+}\right]\left[\mathrm{PPhPy}_{2}\right] \\
\mathrm{Fp}^{+}+\mathrm{PPy}_{3}=\mathrm{FpPPy}_{3}{ }^{+} & K_{4}=\left[\mathrm{FpPPy}_{3}^{+}\right] /\left[\mathrm{Fp}^{+}\right]\left[\mathrm{PPy}_{3}\right]
\end{array}
$$

Hence, the $K_{i j}$ 's listed in Table 1 could be factored, i.e., $K_{12}=K_{2} / K_{1}, K_{13}=K_{3} / K_{1}$, etc. As $\mathrm{Fp}^{+}$is only loosely defined and $\mathrm{PPh}_{3}$ is the most used triarylphosphine, $K_{1}$ could reasonably assume to be unitary in this $\mathrm{Fp}^{+}$-affinity scale to quantify the parameter. Then $K_{2}=4.90, K_{3}=11.0$, and $K_{4}=20.3$.

The incoming phosphine replacement for $\mathrm{CO}$ is generally more difficult than the replacement for phosphine in view of a stronger $\mathrm{Fe}-\mathrm{C}(\mathrm{CO})$ bond than $\mathrm{Fe}-\mathrm{P}$. In the present radical phosphine exchange reactions, the substitution by excess $\mathrm{PPh}_{n} \mathrm{Py}_{3-n}$ for $\mathrm{CO}$ was not observed. We believe that the large cone angles of $\mathrm{PPh}_{n} \mathrm{Py}_{3-n}$ are important here because in the $17 \mathrm{e}-19 \mathrm{e}$ pathways, the reaction rate is very sensitive to steric hindrance. For phosphines of smaller cone angles, e.g., $\mathrm{PPh}_{2} \mathrm{Me}\left(\Theta=136^{\circ}\right)$, we have observed that the phosphine exchange experiment starting $\mathrm{PPh}_{2} \mathrm{Me}$ and $\left[\left(\eta^{5}-\mathrm{C}_{5} \mathrm{H}_{5}\right) \mathrm{Fe}(\mathrm{CO})_{2} \mathrm{PPh}_{3}{ }^{+}\right]$as initialized with a catalytic amount of $\left(\eta^{5}-\mathrm{C}_{5} \mathrm{H}_{5}\right)_{2} \mathrm{Co}$, yielded both $\left[\left(\eta^{5}-\mathrm{C}_{5} \mathrm{H}_{5}\right)-\right.$ $\left.\mathrm{Fe}(\mathrm{CO})_{2} \mathrm{PPh}_{2} \mathrm{Me}^{+}\right]$and $\left[\left(\eta^{5}-\mathrm{C}_{5} \mathrm{H}_{5}\right) \mathrm{Fe}(\mathrm{CO})\left(\mathrm{PPh}_{2} \mathrm{Me}\right)_{2}{ }^{+}\right]$ as products [29]. The double replacement product could be reduced at lower temperatures. A study is now underway in a hope to extend the Fp ${ }^{+}$-affinity scale to $\mathrm{PPh}_{2} \mathrm{Me}$ and/or phosphines of smaller cone angles.

\section{Conclusion}

Application of a radical process results in the rapid phosphine exchange between the $\left[\left(\eta^{5}-\mathrm{C}_{5} \mathrm{H}_{5}\right) \mathrm{Fe}(\mathrm{CO})_{2} \mathrm{PAr}_{3}{ }^{+}\right]$ complexes and the $\mathrm{PR}_{3}$ ligands $\left(\mathrm{PAr}_{3}, \mathrm{PR}_{3}=\mathrm{PPh}_{n} \mathrm{Py}_{3-n}\right.$, $n=0-3$ ) where the donor capability increases in the order $\mathrm{PPh}_{3}<\mathrm{PPh}_{2} \mathrm{Py}<\mathrm{PPhPy}_{2}<\mathrm{PPy}_{3}$ with quantified $\mathrm{Fp}^{+}$affinity scales at $1,4.90,11.0$, and 20.3 , respectively.

\section{Acknowledgements}

Thanks are due to the National Science Council and Academia Sinica for the kind financial support. 


\section{References}

[1] E.C. Alyea, D.W. Meek (Eds.), Catalytic Aspects of Metal Phosphine Complexes, Advances in Chemistry Series 196, American Chemical Society, Washington, DC, 1982.

[2] M.E. Cucullu, C. Li, S.P. Nolan, S.T. Nguyen, R.H. Grubbs, Organometallics 17 (1998) 5565.

[3] C.A. Tolman, Chem. Rev. 77 (1977) 313.

[4] (a) C.A. Streuli, Anal. Chem. 31 (1959) 1652;

(b) W.A. Henderson, C.A. Streuli, J. Am. Chem. Soc. 82 (1960) 5791;

(c) T. Allman, R.G. Goel, Can. J. Chem. 60 (1982) 716;

(d) R.A. Jackson, R. Kanluen, A. Poe, Inorg. Chem. 23 (1984) 523.

[5] (a) T. Bartik, T. Himmler, H.-G. Schulte, K. Seevogel, J. Organomet. Chem. 272 (1984) 29;

(b) C.A. Tolman, J. Am. Chem. Soc. 92 (1970) 2953;

(c) R.G. Goel, W.P. Henry, R.C. Srivastava, Inorg. Chem. 20 (1981) 1727 ;

(d) G.M. Gray, C.S. Kraihanzel, J. Organomet. Chem. 241 (1983) 201;

(e) P.M. Treichel, Inorg. Chem. 7 (1968) 1942;

(f) W.A.G. Graham, Inorg. Chem. 7 (1968) 315;

(g) J.O. Albright, F.L. Tanzella, J.G. Verkade, J. Coord. Chem. 6 (1976) 225;

(h) O. Stelzer, E. Unger, Chem. Ber. 108 (1975) 1246;

(i) C. Barbeau, J. Turcotte, Can. J. Chem. 54 (1976) 1603;

(j) A. Kececi, D. Rehder, Z. Naturforsch. B 36 (1981) 20;

(k) R. Uson, V. Riera, J. Gimeno, M. Laguna, M.P. Gamasa, J. Chem.

Soc., Dalton Trans. (1979) 996;

(1) K. Ihmels, D. Redher, Organometallics 4 (1985) 1334;

(m) P.S. Braterman, D.W. Milne, E.W. Randall, E. Rosenberg, J. Chem. Soc., Dalton Trans. (1973) 1027;

(n) B.E. Mann, C. Masters, B.L. Shaw, J. Chem. Soc. (1971) 1104;

(o) J.A. Timney, Inorg. Chem. 18 (1979) 2502;

(p) S. Vastag, B. Heil, L. Marko, J. Mol. Catal. 5 (1979) 189.

[6] (a) G.M. Bodner, C. Gagnon, D.N. Whittern, J. Organomet. Chem. 243 (1983) 305;

(b) G.M. Bodner, M.P. May, L.E. Mckinney, Inorg. Chem. 19 (1980) 1951;

(c) G.M. Bodner, Inorg. Chem. 14 (1975) 2694;

(d) G.M. Bodner, Inorg. Chem. 13 (1974) 2563;

(e) G.M. Bodner, L.J. Todd, Inorg. Chem. 13 (1974) 1335;

(f) G.M. Bodner, Inorg. Chem. 14 (1975) 1932;

(g) S.O. Grim, R.M. Singer, J. Coord. Chem. 8 (1978) 121;

(h) A.F. Masters, G.E. Bossard, T.A. George, R.T.C. Brownlee,

M.J. O'Connor, A.G. Wedd, Inorg. Chem. 22 (1983) 908;

(i) R.J. Blau, J.H. Espenson, Inorg. Chem. 25 (1986) 878;

(j) R.V. Honeychuck, W.H. Hersh, Inorg. Chem. 26 (1987) 1826;

(k) T.T. Derencsenyi, Inorg. Chem. 20 (1981) 665;

(1) W.C. Trogler, L.G. Marzilli, Inorg. Chem. 14 (1975) 2942;

(m) E.C. Alyea, R.E. Lenkinski, A. Somogyvari, Polyhedron 1 (1982) 130 ;

(n) R.W. Balk, D.J. Stufkens, A. Oskam, Inorg. Chim. Acta 28 (1978) 133.

[7] F.A. Cotton, W.T. Edwards, F.C. Rauch, M.A. Graham, R.N. Perutz, J.J. Turner, J. Coord. Chem. 2 (1973) 247.

[8] (a) B.E. Bursten, D.J. Darensbourg, C.E. Kellog, D.L. Lichtenberger, Inorg. Chem. 23 (1984) 4361;

(b) J.W. Yarbrough, M.B. Hall, Inorg. Chem. 17 (1978) 2269;

(c) R.J. Puddephalt, L. Dignard-Bailey, G.M. Bancroft, Inorg. Chim. Acta 96 (1985) L91;

(d) S. Ikuta, P. Kebarle, G.M.T. Bancroft, R.J. ChanPuddephatt, J. Am. Chem. Soc. 104 (1982) 5899.

[9] (a) B.V. Johnson, A.L. Steinmetz, P.J. Ouseph, J. Coord. Chem. 14 (1985) 103 ;

(b) H. Inoue, M. Sasagawa, E. Fluck, Z. Naturforsch. B 40 (1985) 22; (c) W.E. Carroll, F.A. Deeney, J.A. Delaney, F.J. Lalor, J. Chem. Soc., Dalton Trans. (1973) 718

[10] (a) D.S. Marynick, J. Am. Chem. Soc. 106 (1984) 4064;

(b) M.-H. Whangbo, K.R. Steward, Inorg. Chem. 21 (1982) 1720; (c) S. Xiaxo, W.C. Trogler, D.E. Ellis, Z. Berkovitch-Yellin, J. Am. Chem. Soc. 105 (1983) 7033.

[11] (a) A.M. Bond, S.W. Carr, R. Colton, D.P. Kelly, Inorg. Chem. 22 (1983) 989 ;

(b) D.J. Kuchynka, C. Amatore, J.K. Kochi, Inorg. Chem. 25 (1986) 4087;

(c) J.W. Hershberger, J.K. Kochi, Polyhedron 2 (1983) 929;

(d) C.W. Smith, G.W. Vanloon, M.C. Baird, J. Coord. Chem. 6 (1976) 89;

(e) N.G. Connelly, M.D. Kitchen, J. Chem. Soc., Dalton Trans. (1977) 931

[12] (a) A.G. Orpen, N.G. Connelly, J. Chem. Soc., Chem. Commun. (1985) 1310;

(b) M.J. Wovkulich, J.L. Atwood, L. Canada, J.D. Atwood, Organometallics 4 (1985) 867;

(c) F.A. Cotton, D.J. Darensbourg, S. Klein, B.W.S. Kolthammer, Inorg. Chem. 21 (1982) 294;

(d) F.A. Cotton, D.J. Darensbourg, S. Klein, B.W.S. Kolthammer, Inorg. Chem. 20 (1981) 2661;

(e) N. Bresciani-Pahor, M. Forcolin, L.G. Marzilli, L. Randaccio, M.F. Summers, P.J. Toscano, Coord. Chem. Rev. 63 (1985) 1;

(f) F.A. Cotton, D.J. Darensbourg, W.H. Ilsley, Inorg. Chem. 20 (1981) 578 ;

(g) E.C. Alyea, C. Ferguson, A. Somogyvari, Inorg. Chem. 21 (1982) 1369 ;

(h) F.A. Cotton, D.J. Darensbourg, B.W.S. Kolthammer, Inorg. Chem. 20 (1981) 4440.

[13] M. Rahman, Md.Y.-Y. Liu, K. Eriks, A. Prock, W.P. Giering, Organometallics 8 (1989) 1.

[14] R.L. Rich, H. Taube, J. Am. Chem. Soc. 76 (1954) 2608

[15] W.C. Trogler (Ed.), Organometqallic Radical Processes, Elsevier, Amsterdam, 1990.

[16] (a) D.R. Tyler, Prog. Inorg. Chem. 36 (1988) 125;

(b) D. Astruc, Chem. Rev. 88 (1988) 1189;

(c) M.C. Baird, Chem. Rev. 88 (1990) 1217;

(d) D.R. Tyler, F. Mao, Coord. Chem. Rev. 97 (1990) 119;

(e) D.R. Tyler, Acc. Chem. Rev. 24 (1991) 325.

[17] D. Astruc, Electron Transfer and Radical Process in Transition Metal Chemistry, VCH, Weinheim, 1995.

[18] K.A. Pevear, M.M. Banaszak Holl, G.B. Carpenter, A.L. Rieger, P.H. Rieger, D.A. Stweigart, Organometallics 14 (1995) 512.

[19] (a) M.Y. Darensbourg, J. Organomet. Chem. 38 (1972) 133; (b) E.C. Ashby, T.N. Pham, B. Park, Tetrahedron Lett. 26 (1985) 4691 ;

(c) H. Yamataka, N. Fujimura, Y. Kawafujim, T. Hanafusa, J. Am. Chem. Soc. 109 (1987) 4305.

[20] S.L. Gibson, L.-K. Liu, R.U. Soliz, J. Organomet. Chem. 526 (1996) 393.

[21] D.D. Perrin, W.L.F. Armarego, D.R. Perrin, Purification of Laboratory Chemicals, Pergamon Press, Oxford, 1981.

[22] (a) B.D. Dombek, R.J. Angelici, Inorg. Chim. Acta 7 (1973) 345; (b) T.J. Meyer, E.C. Johnson, N. Winterton, Inorg. Chem. 10 (1971) 1673 ;

(c) T.J. Meyer, E.C. Johnson, N. Winterton, Inorg. Synth. 12 (1971) 36 ;

(d) T.J. Meyer, E.C. Johnson, N. Winterton, Inorg. Synth. 7 (1963) 110

(e) R.B. King, M.B. Bisnette, J. Organomet. Chem. 2 (1964) 15.

[23] T.S. Piper, G. Wilkinson, J. Inorg. Nucl. Chem. 3 (1956) 104.

[24] A. Maisonnet, J.P. Farr, M.M. Olmstead, C.T. Hunt, Inorg. Chem. 21 (1982) 3961.

[25] (a) F.G. Mann, J. Watson, J. Org. Chem. 13 (1948) 502;

(b) K. Kurtev, D. Ribola, R.A. Jones, D.J. Cole-Hamilton, G. Wilkinson, J. Chem. Soc., Dalton Trans. (1980) 56.

[26] P.M. Treichel, R.L. Shubkin, Inorg. Chem. 6 (1967) 1328.

[27] J.P. Bibler, A. Wojcicki, Inorg. Chem. 5 (1966) 889.

[28] L.-S. Luh, U.B. Eke, L.-K. Liu, Organometallics 14 (1995) 440.

[29] J. Peng, L.-K. Liu, Bull. Inst. Chem., Acad. Sin. 45 (1998) 27. 